\title{
Bullous Retiform Purpura on the Ears and Legs
}

\author{
Shane M. Swink, DO, MS; Naeha Gupta, DO; Haider Asad, MD; Claire Dorfman, DO; \\ Nektarios Lountzis, MD
}

\section{Eligible for 1 MOC SA Credit From the ABD}

This Dermatopathology Diagnosis in our print edition is eligible for 1 self-assessment credit for Maintenance of Certification from the American Board of Dermatology (ABD). After completing this activity, diplomates can visit the ABD website (http://www.abderm.org) to self-report the credits under the activity title "Cutis Dermatopathology Diagnosis." You may report the credit after each activity is completed or after accumulating multiple credits.

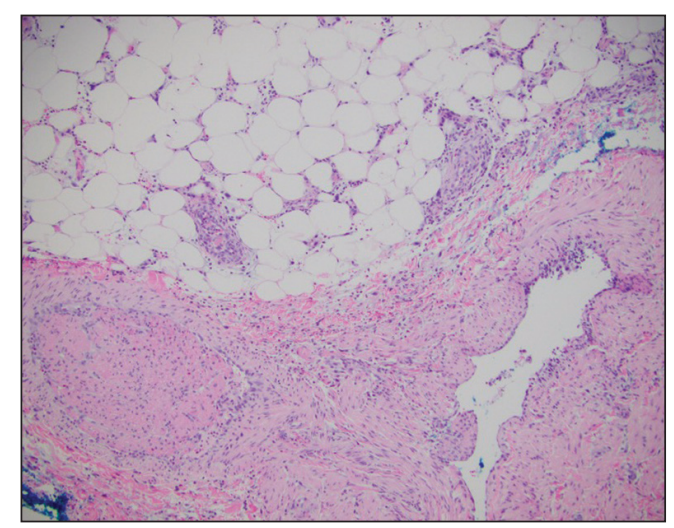

H\&E, original magnification $\times 100$.

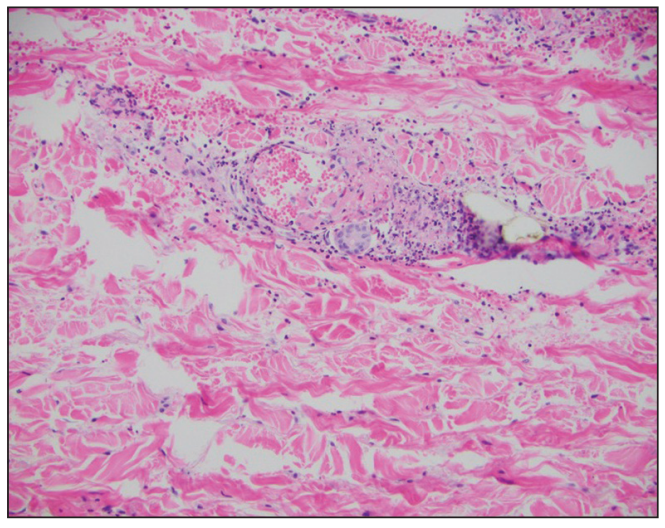

H\&E, original magnification $\times 200$.
A 40-year-old woman presented with a progressive painful rash on the ears and legs of 2 weeks' duration. She described the rash as initially red and nonpainful; it started on the right leg and progressed to the left leg, eventually involving the earlobes 4 days prior to presentation. Physical examination revealed edematous purpura of the earlobes and bullous retiform purpura on the lower extremities. Laboratory studies revealed leukopenia $\left(3.6 \times 10^{3} / \mathrm{cm}^{2}\right.$ [reference range, $\left.\left.4.0-10.5 \times 10^{3} / \mathrm{cm}^{2}\right]\right)$ and elevated antineutrophil cytoplasmic antibodies (1:320 titer [reference range, <1:40]) in a perinuclear pattern (perinuclear antineutrophil cytoplasmic antibodies). Urine toxicology screening was positive for cocaine and opiates. A punch biopsy of a bullous retiform purpura on the right thigh was obtained for standard hematoxylin and eosin staining.

\section{THE BEST DIAGNOSIS IS:}
a. ecthyma gangrenosum
b. eosinophilic granulomatosis with polyangiitis
c. idiopathic thrombocytopenic purpura
d. levamisole-induced vasculopathy
e. polyarteritis nodosa

PLEASE TURN TO PAGE 97 FOR THE DIAGNOSIS

Drs. Swink, Gupta, Asad, and Dorfman are from Lehigh Valley Health Network, Allentown, Pennsylvania. Dr. Lountzis is from Advanced Dermatology Associates, LTD, Allentown.

The authors report no conflict of interest.

Correspondence: Shane M. Swink, DO, MS, 1259 S Cedar Crest Blvd, Ste 100, Allentown, PA 18103 (shanesw@pcom.edu). doi:10.12788/cutis.0322 


\section{THE DIAGNOSIS:}

\section{Levamisole-Induced Vasculopathy}

P iopsy of one of the bullous retiform purpura on the leg (Figure 1) revealed a combined leukocytoclastic vasculitis and thrombotic vasculopathy (quiz images). Periodic acid-Schiff and Gram stains, with adequate controls, were negative for pathogenic fungal and bacterial organisms. Although this reaction pattern has an extensive differential, in this clinical setting with associated cocainepositive urine toxicologic analysis, perinuclear antineutrophil cytoplasmic antibodies (p-ANCA), and leukopenia, the histopathologic findings were consistent with levamisoleinduced vasculopathy (LIV). ${ }^{1,2}$ Although not specific, leukocytoclastic vasculitis and thrombotic vasculopathy have been reported as the classic histopathologic findings of LIV. In addition, interstitial and perivascular neovascularization have been reported as a potential histopathologic finding associated with this entity but was not seen in our case. ${ }^{3}$

Levamisole is an anthelminthic agent used to adulterate cocaine, a practice first noted in 2003 with increasing incidence. ${ }^{1}$ Both levamisole and cocaine stimulate the sympathetic nervous system by increasing dopamine in the euphoric areas of the brain. ${ }^{1,3}$ By combining the 2 substances, preparation costs are reduced and stimulant effects are enhanced. It is estimated that $69 \%$ to $80 \%$ of cocaine in the United States is contaminated with levamisole. .,4,5 $^{2,5}$ The constellation of findings seen in patients abusing levamisole-contaminated cocaine include agranulocytosis; p-ANCA; and a tender, vasculitic, retiform purpura presentation. The most common sites for the purpura include the cheeks and ears. The purpura can progress to bullous lesions, as seen in our patient, followed by necrosis., ${ }^{4,6}$ Recurrent use of levamisole-contaminated cocaine is associated with recurrent agranulocytosis and classic skin findings, which is suggestive of a causal relationship. ${ }^{6}$

Serologic testing for levamisole exposure presents a challenge. The half-life of levamisole is relatively short (estimated at 5.6 hours) and is found in urine samples approximately $3 \%$ of the time. ${ }^{1,3,6}$ The volatile diagnostic characteristics of levamisole make concrete laboratory confirmation difficult. Although a skin biopsy can be helpful to
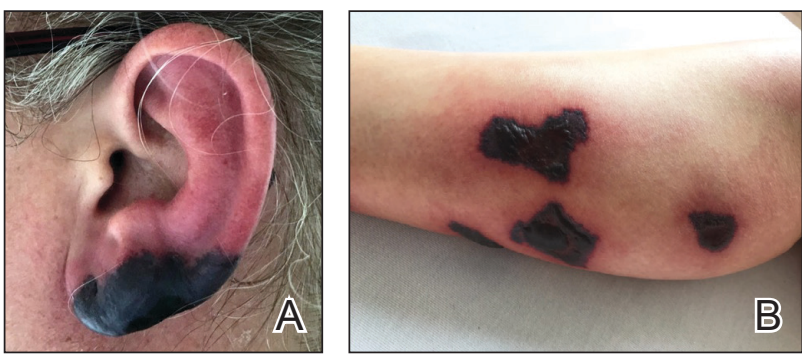

FIGURE 1. $A$ and B, Edematous purpura of the earlobe and bullous retiform purpura on the leg. rule out other causes of vasculitislike presentations, it is not specific for LIV. Therefore, clinical suspicion for LIV should remain high in patients who present with the cutaneous findings described as well as agranulocytosis, positive p-ANCA, and a history of cocaine use with a skin biopsy showing leukocytoclastic vasculitis and thrombotic vasculopathy.

The differential diagnosis for LIV with retiform bullous lesions includes several other vasculitides and vesiculobullous diseases. Eosinophilic granulomatosis with polyangiitis (EGPA) is a multisystem vasculitis that is characterized by eosinophilia, asthma, and rhinosinusitis. Eosinophilic granulomatosis with polyangiitis primarily affects small and medium arteries in the skin and respiratory tract and occurs in 3 stages: prodromal, eosinophilic, and vasculitic. These stages are characterized by mild asthma or rhinitis, eosinophilia with multiorgan infiltration, and vasculitis with extravascular granulomatosis, respectively. Diagnosis often is clinical based on these findings and laboratory evaluation. Eosinophilic granulomatosis with polyangiitis presents with positive p-ANCA in $40 \%$ to $60 \%$ of patients. ${ }^{7}$ The vasculitis stage of EGPA presents with cutaneous findings in $60 \%$ of cases, including palpable purpura, infiltrated papules and plaques, urticaria, necrotizing lesions, and rarely vesicles and bullae. ${ }^{8}$ Classic histopathologic features include leukocytoclastic or eosinophilic vasculitis, an eosinophilic infiltrate, granuloma formation, and eosinophilic granule deposition onto collagen fibrils (otherwise known as flame figures) (Figure 2). Biopsy of these lesions with the aforementioned findings, in constellation with the described systemic signs and symptoms, can aid in diagnosis of EGPA.

Polyarteritis nodosa (PAN) is a vasculitis that can be either multisystem or limited to one organ. Classic PAN affects the small- to medium-sized vessels. When there is multisystem involvement, it most often affects the skin, gastrointestinal tract, and kidneys. It presents with subcutaneous or dermal nodules, necrotic lesions, livedo reticularis, hypertension, abdominal pain, and an acute abdomen. ${ }^{9}$

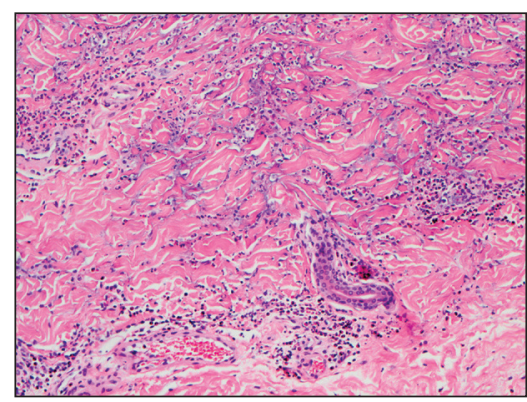

FIGURE 2. Eosinophilic granulomatosis with polyangiitis. Leukocytoclastic vasculitis with a dermal eosinophilic infiltrate and eosinophilic granules deposited onto collagen fibrils (H\&E, original magnification $\times 200$ ). 
When PAN is in its limited form, it most commonly occurs in the skin. The cutaneous manifestations of skin-limited PAN are identical to classic PAN, most commonly occurring on the legs and arms and less often on the trunk, head, and neck. ${ }^{10}$ To aid in diagnosis, biopsies of cutaneous lesions are beneficial. Dermatopathologic examination of PAN reveals fibrinoid necrosis of small and medium vessels with a perivascular mononuclear inflammatory infiltrate (Figure 3). Cutaneous PAN rarely progresses to multisystem classic PAN and carries a more favorable prognosis.

Microvascular occlusion syndromes can result in clinical presentations that resemble LIV. Idiopathic thrombocytopenic purpura is a hematologic autoimmune condition

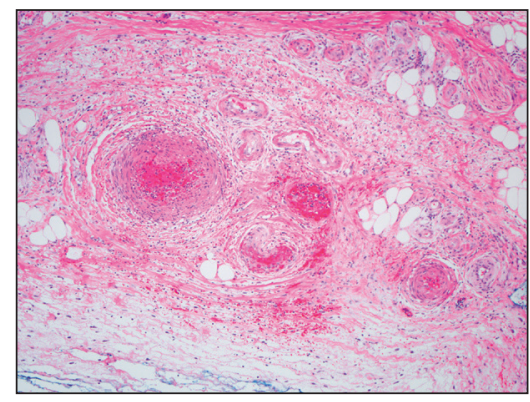

FIGURE 3. Polyarteritis nodosa. Fibrinoid necrosis of small and medium vessels with a perivascular mononuclear inflammatory infiltrate (H\&E, original magnification $\times 200)$.
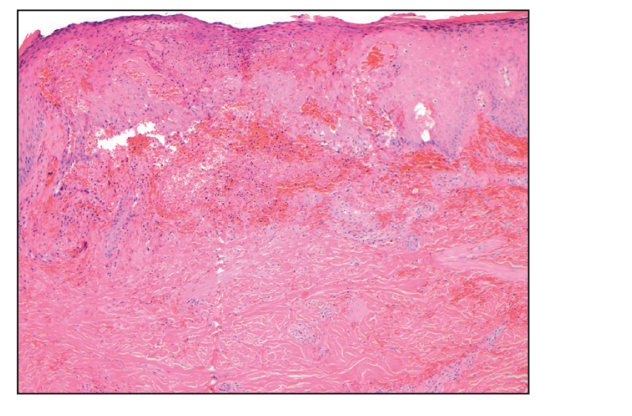

FIGURE 4. Idiopathic thrombocytopenic purpura. Dermal and epidermal extravasation of red blood cells with incipient hemorrhagic intraepidermal bullae (H\&E, original magnification $\times 100)$.

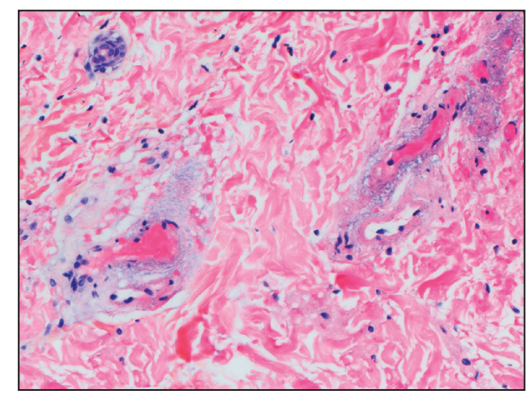

FIGURE 5. Ecthyma gangrenosum. Dilated and congested dermal blood vessels. Basophilic granular material is seen surrounding the vasculature with a slight mononuclear inflammatory infiltrate (H\&E, original magnification $\times 400$ ) resulting in destruction of platelets and subsequent thrombocytopenia. Idiopathic thrombocytopenic purpura can be either primary or secondary to infections, drugs, malignancy, or other autoimmune conditions. Clinically, it presents as mucosal or cutaneous bleeding, epistaxis, hematochezia, or hematuria and can result in substantial hemorrhage. On the skin, it can appear as petechiae and ecchymoses in dependent areas and rarely hemorrhagic bullae of the skin and mucous membranes in cases of severe thrombocytopenia. ${ }^{11,12}$ Biopsies of these lesions will show notable extravasation of red blood cells with incipient hemorrhagic bullae formation (Figure 4). Recognition of hemorrhagic bullae as a presentation of idiopathic thrombocytopenic purpura is critical to identifying severe underlying disease.

Beyond other vasculitides and microvascular occlusion syndromes, vessel-invasive microorganisms can result in similar histopathologic and clinical presentations to LIV. Ecthyma gangrenosum (EG) is a septic vasculitis, often caused by Pseudomonas, aeruginosa, usually affecting immunocompromised patients. Ecthyma gangrenosum presents with vesiculobullous lesions with erythematous violaceous borders that develop into hemorrhagic bullae with necrotic centers. ${ }^{13}$ Biopsy of EG will show vascular occlusion and basophilic granular material within or around vessels, suggestive of bacterial sepsis (Figure 5). The detection of an infectious agent on histopathology allows one to easily distinguish between EG and LIV.

\section{REFERENCES}

1. Bajaj S, Hibler B, Rossi A. Painful violaceous purpura on a 44-year-old woman. Am J Med. 2016;129:E5-E7.

2. Munoz-Vahos CH, Herrera-Uribe S, Arbelaez-Cortes A, et al. Clinical profile of levamisole-adulterated cocaine-induced vasculitis/vasculopathy. J Clin Rheumatol. 2019;25:E16-E26.

3. Jacob RS, Silva CY, Powers JG, et al. Levamisole-induced vasculopathy: a report of 2 cases and a novel histopathologic finding. Am J Dermatopathol. 2012;34:208-213.

4. Gillis JA, Green P, Williams J. Levamisole-induced vasculopathy: staging and management. J Plast Reconstr Aesthet Surg. 2014;67:E29-E31.

5. Farhat EK, Muirhead TT, Chafins ML, et al. Levamisole-induced cutaneous necrosis mimicking coagulopathy. Arch Dermatol. 2010;146:1320-1321.

6. Chung C, Tumeh PC, Birnbaum R, et al. Characteristic purpura of the ears, vasculitis, and neutropenia-a potential public health epidemic associated with levamisole-adulterated cocaine. J Am Acad Dermatol. 2010;65:722-725

7. Negbenebor NA, Khalifian S, Foreman RK, et al. A 92-year-old male with eosinophilic asthma presenting with recurrent palpable purpuric plaques. Dermatopathology (Basel). 2018;5:44-48.

8. Sherman S, Gal N, Didkovsky E, et al. Eosinophilic granulomatosis with polyangiitis (Churg-Strauss) relapsing as bullous eruption. Acta Derm Venereol. 2017;97:406-407.

9. Braungart S, Campbell A, Besarovic S. Atypical Henoch-Schonlein purpura? consider polyarteritis nodosa! BMJ Case Rep. 2014. doi:10.1136/bcr-2013-201764

10. Alquorain NAA, Aljabr ASH, Alghamdi NJ. Cutaneous polyarteritis nodosa treated with pentoxifylline and clobetasol propionate: a case report. Saudi J Med Sci. 2018;6:104-107.

11. Helms AE, Schaffer RI. Idiopathic thrombocytopenic purpura with black oral mucosal lesions. Cutis. 2007;79:456-458.

12. Lountzis N, Maroon M, Tyler W. Mucocutaneous hemorrhagic bullae in idiopathic thrombocytopenic purpura. J Am Acad Dermatol. 2009;60:AB124.

13. Llamas-Velasco M, AlegeriaV, Santos-Briz A, et al. Occlusive nonvasculitic vasculopathy. Am J Dermatopathol. 2017;39:637-662. 